\title{
Fluid drop shape determination by the Rayleigh-Ritz minimization method
}

\author{
F. P.-A. Cortat* $\quad$ S. J. Miklavcic*
}

(Received 16 March 2006, revised 15 April 2007)

\begin{abstract}
The Rayleigh-Ritz (RR) method is well known as a means of minimizing energy functionals. Despite this, the technique most often employed in practice for minimizing a functional is the numerical solution of the Euler-Lagrange (EL) equations derived from the energy functional by variational minimization. In this article we employ the $R R$ method specifically to determine the equilibrium shape of a fluid drop interface deformed by externally applied surface stresses and compare the results with numerical solution of the EL equations. We give examples of conditions where the RR method is superior in terms of simplicity and accuracy to the numerical EL solution, as well as conditions under which the method is less reliable.
\end{abstract}

*Department of Science and Technology, Linköping University, Campus Norrköping, S-601 74, Norrköping, Sweden. mailto:freco@itn.liu.se

See http://anziamj . austms.org.au/V47EMAC2005/Cortat1 for this article, (C) Austral. Mathematical Soc. 2007. Published June 26, 2007. ISSN 1446-8735 


\section{Contents}

1 Introduction

C777

2 Free energy of sessile drop under gravitational and arbitrary surface stress

C778

3 Rayleigh-Ritz determination of sessile drop shapes $\quad$ C781 3.1 Incorporation of physical constraints . . . . . . . . . C781

3.2 Energy functional minimization . . . . . . . . . . . C783

3.3 Choice of basis functions . . . . . . . . . . . . C785

4 Numerical results and discussion $\quad \mathbf{C 7 8 5}$

4.1 An unstressed sessile drop . . . . . . . . . . . . . C786

4.2 A sessile drop stressed under gravity . . . . . . . . . C792

4.3 A sessile drop stressed under gravity and interaction . . . . C795

5 Overall summary

C800

References

C800

\section{Introduction}

During the past few years there has been growing experimental $[1,2,3$, $4,5,6,7,8,9,10,11]$ and theoretical $[12,13,14,15,16,17,18,19,20$, 21, 22], interest in systems where deformable surfaces interact with colloidal particles in liquids. These systems are important as they commonly appear in both nature and in technical situations. Practical examples arise in areas such as biotechnology (involving biological cells, vesicles, emulsions and the like) and in the pulp and paper industry (for example, with the de-inking of recycled paper fibres by flotation). The focus of study is on events related to the interaction between free surfaces of bubbles and other fluid drops 
and solid particles. One core property that must be established for any quantitative assessment of the effects of interaction is the deformed shape that the drop adopts. Determination of this shape, through solution of a non-linear problem, must invariably be achieved numerically. However, it is desirable to have a simpler, more expedient, though equally accurate means to determine this quantity. One aim of our continued efforts is the derivation of a simple, robust and general approach to modeling the shape behavior of liquid drops as they are stressed by colloidal or other influences.

Here we consider an alternate simple, semi-analytical method and use it to address a number of free surface problems. Instead of following the traditional route of numerically solving the Euler-Lagrange equation for this problem, or deriving approximate solutions of this equation [21, 17, 22], we tackle the energy minimization directly by means of the Rayleigh-Ritz method [23]. The desired equilibrium solution is expressed as a sum/series of appropriately defined basis functions. The series involves a number of free parameters which are subsequently determined by the condition that they collectively minimize the free energy functional for the problem. For three different problems of increasing complexity, we compare the results of this direct approach against results of the more traditional numerical line (described in Section 2) and discuss its advantages and disadvantages.

\section{Free energy of sessile drop under gravitational and arbitrary surface stress}

The system we consider consists of a fluid drop, deposited on a solid substrate and interacting with a spherical particle via a given, attractive force law (see Figure 1a). The system is assumed axisymmetric. That is, we assume cylindrical symmetry around the $z$-axis, defined to be perpendicular to the substrate and extending through the apex of the drop. Axisymmetry implies that the particle is positioned exactly above the apex. Furthermore, the 

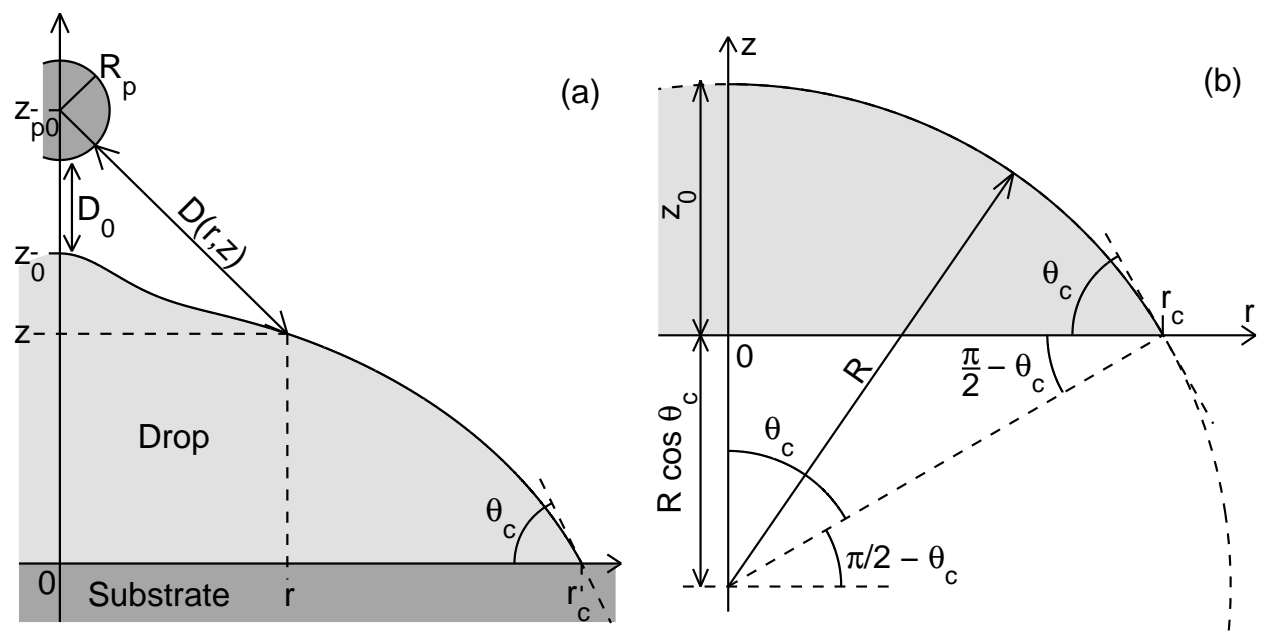

Figure 1: (a) Schematic of the drop-particle interaction system. The extent of the drop deformation due to the interaction with the spherical particle is deliberately exaggerated. (b) Schematic of the unstressed sessile drop. The geometry of the drop profile corresponds to a part of a sphere. 
contact angle, which the drop makes with the substrate is assumed strictly smaller than $90^{\circ}$; thus we describe the drop shape by specifying its height, $z$, as a function of $r$, the radius.

The free energy, $\mathcal{F}$, as a functional of the shape, $z(r)$, involves terms from three physical contributions: a surface energy describing the work done in creating the surface, proportional to the surface tension, $\gamma$; a local surface stress, $\sigma(r, z)$, acting over the entire surface arising from the interaction between the free interface and another body; and a buoyancy term proportional to the gravitational acceleration, $g$, and the density difference, $\Delta \rho$. Finally, assuming incompressibility, the condition of constant volume is enforced by the fourth term involving a Lagrangian multiplier, $\Lambda$. Under a constant contact radius, $r_{c}$, assumption, the free energy functional is

$$
\mathcal{F}=2 \pi \int_{0}^{r_{c}} r\left[(\gamma+\sigma) \sqrt{1+z_{r}^{2}}+\frac{g \Delta \rho}{2} z^{2}+\Lambda z\right] d r,
$$

where $z_{r}:=\partial z / \partial r$. By minimizing this expression we determine the profile shape function, $z(r)$.

To minimize the free energy (1) in the domain of admissible functions, a subset of the set of non-negative functions over the interval $\left[0, r_{c}\right]$ is used. The admissible functions must be twice differentiable in $\left[0, r_{c}\right]$, have zero slope at the apex, $\left.z_{r}\right|_{r=0}=0$, from cylindrical symmetry, and $r_{c}$ must be their first zero. The Euler-Lagrange variational method applied to equation (1) gives [20]

$$
(\gamma+\sigma)\left(\frac{z_{r}}{\sqrt{1+z_{r}^{2}}}+\frac{r z_{r r}}{\left(1+z_{r}^{2}\right)^{3 / 2}}\right)+\frac{r}{\sqrt{1+z_{r}^{2}}}\left(\sigma_{r} z_{r}-\sigma_{z}\right)=r(g \Delta \rho z+\Lambda) .
$$

This is a second order non-linear differential equation for $z(r)$, involving both gravity and surface energy density. This equation has not been solved analytically; accurate numerical solutions are really the only general recourse.

The surface energy density, $\gamma+\sigma$, is generally a complicated function 
of $z(r)$. However, we simplify matters by taking $\sigma$ as a function of the single quantity, $D(r, z)$, being the perpendicular distance between the particle and the profile (see Figure 1). $\sigma(D)$ corresponds to the work done in bringing the particle from infinity to a distance $D$, against a pressure field, $\pi$, due to the interaction. Taking $\pi(D)$ as the pressure field between two infinite plates, we have

$$
\sigma(D)=-\int_{\infty}^{D} \pi\left(D^{\prime}\right) d D^{\prime} \quad \text { where } \quad \pi(D)=-\frac{\mathcal{A}}{6 \pi D^{3}} .
$$

$\mathcal{A}$ is a measure of the strength of the van der Waals surface interaction, taking values in the range $\left[2 \cdot 10^{-22}, 2 \cdot 10^{-20}\right] \mathrm{J}$. The perpendicular distance between the particle and the profile shape, $D(r, z)$ (see Figure 1a), is defined by the coordinate pair $\{r, z(r)\}:\left(D+R_{p}\right)^{2}=r^{2}+\left(z_{p 0}-z\right)^{2}$. Here, $R_{p}$ is the radius of the spherical particle and $z_{p 0}$ denotes the height of its center above the reference level $z=0$ (substrate). By the above integral, the effective local interaction energy is

$$
\sigma(r, z(r))=-\frac{\mathcal{A}}{12 \pi}\left(\sqrt{\left(z_{p 0}-z(r)\right)^{2}+r^{2}}-R_{p}\right)^{-2} .
$$

\section{Rayleigh-Ritz determination of sessile drop shapes}

The approximate solution we discuss in this section is based on the RayleighRitz method, a technique that tackles the minimization or variation problems directly [23].

\subsection{Incorporation of physical constraints}

A number of conditions that must be satisfied by the equilibrium shape are incorporated directly into the definition of basis functions. The first is 
the constraint of constant volume. We begin with an ansatz of the form $z(r)=\sum_{n=1}^{N} a_{n} f_{n}(r)$, where $f_{n}(r)$ are arbitrarily chosen functions. The volume condition assuming an axisymmetric drop whose maximum radial extent is a fixed radius $r_{c}$, takes the form

$$
\int_{0}^{r_{c}} r z(r) d r=\int_{0}^{r_{c}} r\left(\sum_{n=1}^{N} a_{n} f_{n}(r)\right) d r=V_{0} .
$$

This represents an equation for $a_{1}$, in terms of the remaining coefficients. Explicitly, we have

$$
a_{1}=\frac{V_{0}}{\int_{0}^{r_{c}} r f_{1}(r) d r}-\sum_{n=2}^{N} a_{n} \frac{\int_{0}^{r_{c}} r f_{n}(r) d r}{\int_{0}^{r_{c}} r f_{1}(r) d r} .
$$

Consequently, the ansatz takes the form

$$
z(r)=a_{1} f_{1}(r)+\sum_{n=2}^{N} a_{n} f_{n}(r)=y_{0}(r)+\sum_{n=2}^{N} a_{n} y_{n}(r),
$$

which explicitly involves the $N-1$ coefficients $a_{2}, \ldots, a_{N}$, and new functions

$$
y_{0}(r):=\frac{V_{0} f_{1}(r)}{\int_{0}^{r_{c}} r f_{1}(r) d r} \quad, \quad y_{n}(r):=-\frac{\int_{0}^{r_{c}} r f_{n}(r) d r}{\int_{0}^{r_{c}} r f_{1}(r) d r} f_{1}(r)+f_{n}(r) .
$$

$y_{0}(r)$ alone explicitly fulfills the volume condition.

A second constraint is one of either of two options: a fixed contact radius, $z\left(r_{c}\right)=0$ and fixed contact angle condition, $z_{r}\left(r_{c}\right)=-\tan \theta_{c}$. In the last case, $\theta_{c}$ is fixed by the relative strengths of surface energies and $r_{c}$ is to be determined. In the first case we have

$$
z\left(r_{c}\right)=0 \quad \Rightarrow \quad \sum_{n=2}^{N} a_{n} y_{n}\left(r_{c}\right)=-y_{0}\left(r_{c}\right) .
$$


For arbitrary basis functions, $f_{n}(r)$, the $y_{n}$ do not necessarily all vanish at $r_{c}$ for all $n$. Hence, the above defines a second equation involving all of the $N-1$ coefficients, which is be solved for, say, $a_{2}$. Provided $y_{2}\left(r_{c}\right) \neq 0$,

$$
a_{2}=-\frac{\sum_{n=3}^{N} a_{n} y_{n}\left(r_{c}\right)+y_{0}\left(r_{c}\right)}{y_{2}\left(r_{c}\right)} .
$$

The second constraint thus leads to the new ansatz

$$
z(r)=y_{0}(r)+a_{2} y_{2}(r)+\sum_{n=3}^{N} a_{n} y_{n}(r)=z_{0}(r)+\sum_{n=3}^{N} a_{n} \phi_{n}(r),
$$

in which we define new functions

$$
z_{0}(r):=y_{0}(r)+a_{2} y_{2}(r), \quad \phi_{n}(r):=-\frac{y_{n}\left(r_{c}\right)}{y_{2}\left(r_{c}\right)} y_{2}(r)+y_{n}(r) .
$$

These functions now satisfy both the constant volume condition and the constant contact radius boundary condition for any initial choice of functions. This process is repeated for each physical constraint one wishes to impose on the desired solution.

\subsection{Energy functional minimization}

Explicit use of (4) in the Rayleigh-Ritz method applied to the free energy functional (1), yields

$$
\begin{aligned}
\mathcal{F}[z(r)] & =2 \pi \int_{0}^{r_{c}} r\left[(\gamma+\sigma(r, z(r))) \sqrt{1+z_{r}^{2}}+\frac{g \Delta \rho}{2} z(r)^{2}\right] d r \\
& =\int_{0}^{r_{c}} f\left(r, \sum_{i=3}^{N} a_{i} \phi_{i}, \sum_{i=3}^{N} a_{i} \phi_{i, r}\right) d r=\mathcal{F}\left(a_{3}, a_{4}, \ldots, a_{N}\right) .
\end{aligned}
$$


Minimization of (6) proceeds through partial differentiation with respect to the parameters $\left\{a_{n}\right\}_{n=3}^{N}$, and results in the system of equations,

$$
\frac{\partial \mathcal{F}}{\partial a_{n}}=0, \quad i=3, \ldots, n .
$$

Noting that $\partial z / \partial a_{n}=\phi_{n}(r)$, the partial derivatives of $\mathcal{F}$ with respect to $\left\{a_{n}\right\}_{n=3}^{N}$ are

$$
\frac{\partial \mathcal{F}}{\partial a_{n}}=2 \pi \int_{0}^{r_{c}} r\left[(\gamma+\sigma) \frac{z_{r}}{\sqrt{1+z_{r}^{2}}} \frac{\partial \phi_{n}}{\partial r}+\sigma_{z} \phi_{n} \sqrt{1+z_{r}^{2}}+g \Delta \rho z \phi_{n}\right] d r,
$$

where the final expansion (4) is inserted for $z(r)$. Equations (7) are solved for $\left\{a_{n}\right\}_{n=3}^{N}$ using the Newton-Raphson method. The multi-dimensional version of the Newton-Raphson routine applied to our given problem is

$$
a_{i+1}=a_{i}-\left[\left.\left(\frac{\overrightarrow{\partial \mathcal{F}}}{\partial a} \cdot J^{-1}\right)\right|_{a_{n}=a_{i}}\right]_{i}
$$

where $\overrightarrow{\partial \mathcal{F} / \partial a}$ is the vector gradient of $\mathcal{F}$ with respect to the $a_{n}$, and $J$ is the Jacobian matrix whose components are the second partial derivatives of the energy, $\mathcal{F}$, with respect to $a_{i}$ and $a_{j}$,

$$
\begin{aligned}
& \frac{\partial^{2} \mathcal{F}}{\partial a_{i} a_{j}}=2 \pi \int_{0}^{r_{c}} r\left[\left(\sigma_{z z} \sqrt{1+z_{r}^{2}}+g \Delta \rho\right) \phi_{i}(r) \phi_{j}(r)\right] d r \\
& +2 \pi \int_{0}^{r_{c}} r\left[\frac{z_{r} \sigma_{z}}{\sqrt{1+z_{r}^{2}}}\left(\phi_{i}(r) \frac{\partial \phi_{j}}{\partial r}+\phi_{j}(r) \frac{\partial \phi_{i}}{\partial r}\right)+\frac{\gamma+\sigma}{\sqrt[3]{1+z_{r}^{2}}} \frac{\partial \phi_{i}}{\partial r} \frac{\partial \phi_{j}}{\partial r}\right] d r .
\end{aligned}
$$

Here and in (8), we used

$$
\frac{\partial \sigma}{\partial a_{n}}=\frac{\partial \sigma}{\partial z} \frac{\partial z}{\partial a_{n}}=\sigma_{z} \phi_{n}(r), \quad \frac{\partial^{2} \sigma}{\partial a_{m} a_{n}}=\frac{\partial^{2} \sigma}{\partial z^{2}} \frac{\partial z}{\partial a_{m}} \frac{\partial z}{\partial a_{n}}=\sigma_{z z} \phi_{n}(r) \phi_{m}(r) .
$$


Actual inversion of the Jacobian and solution for the vector of coefficients $\left\{a_{n}\right\}$ is achieved using a Gaussian elimination scheme. The free energy functional (6), and its various first (8) and second (9) partial derivatives are explicitly given for rapid evaluation. All integrations are numerically calculated using the trapezoidal rule.

\subsection{Choice of basis functions}

The Rayleigh-Ritz method is a fast and manageable method which is, however, dependent on the choice of functions, $f_{n}(r)$. We shall see that an appropriate choice of the $f_{n}(r)$ significantly improves the accuracy of the approximation. In the above discussion and in the numerical implementation, we implicitly assumed the functions, $f_{n}(r)$, satisfy axisymmetry: $\partial f_{n} / \partial r=0$ at $r=0$, and will even satisfy the constraint $f_{n}\left(r_{c}\right)=0$. Since we specified $a_{2}$ in (4), this last requirement is not necessary and we can choose more general functions. However, to ensure as few expansion coefficients as possible and save on computation time, it is easier to choose basis functions that fulfill both these conditions. In the numerical study we implement four different options:

$$
f_{n}(r) \in\left\{r_{c}^{2 n}-r^{2 n}, \quad \cos \left(\frac{\pi n}{2} \frac{r}{r_{c}}\right), \quad \frac{1}{n} e^{-n\left(r / r_{c}\right)^{2}}, \quad J_{0}\left(\alpha_{0 n} \frac{r}{r_{c}}\right)\right\},
$$

referred to as algebraic, trigonometric, exponential and Bessel bases, respectively.

\section{Numerical results and discussion}

We apply the method described above to three different systems of sessile drops. In order of increasing physical complexity, the problems we consider 
are as follows. First is a free standing, unstressed drop of known volume resting on a horizontal substrate. This system alone of the three has an exact, closed form solution for the optimal shape: the minimizing free surface in this case is spherical, which provides us with an analytical test of the Rayleigh-Ritz method. The second problem involves a fluid drop sessile on a horizontal substrate subject only to the body force of gravity. No exact analytical solution is known. For tests of accuracy we refer to the solution obtained by numerical integration of the differential equation (2). Finally, we consider the problem of a fluid drop, sessile under a gravitational body force, interacting through an attractive van der Waals force with a spherical particle of typical colloidal size. The results produced by the Rayleigh-Ritz method are presented for various numbers of terms $(N)$, basis functions, $f_{n}(r)$, and initial profiles, $z_{0}(r)$.

\subsection{An unstressed sessile drop}

It can be verified that the exact solution of the Euler-Lagrange equation for an unstressed sessile drop of given volume, $V_{0}$, is a section of a sphere. Figure $1 \mathrm{~b}$ shows the relevant geometry. The sphere's radius is $R=\left(r_{c}^{2}+\right.$ $\left.z_{0}^{2}\right) / 2 z_{0}$, in terms of maximum height above the substrate, $z_{0}$, and contact radius, $r_{c}$. The volume for that part of the sphere above the substrate, $z=0$, is $V_{0}=\frac{\pi}{6} z_{0}\left(3 r_{c}^{2}+z_{0}^{2}\right)$. Solving this third order equation for $z_{0}$, the only real root is substituted to solve for the sphere radius, $R$. Consequently, the sphere shape is

$$
z_{\mathrm{sph}}(r)=z_{0}-R+\sqrt{R^{2}-r^{2}} .
$$

This exact solution is used for comparison with the Rayleigh-Ritz approximation, (4), using various basis functions, $f_{n}(r)$.

In the systems considered below we have no gravity and no interaction. The physical parameters are then $g \Delta \rho=0, \gamma=72.8 \mathrm{mN} / \mathrm{m}, V_{0}=25 \mu \mathrm{L}$ and $\mathcal{A}=0$. The prescribed drop radius, $r_{c}$, is the only variable we vary in this case and of course the number of terms $N$ involving coefficients, $a_{n}$, 


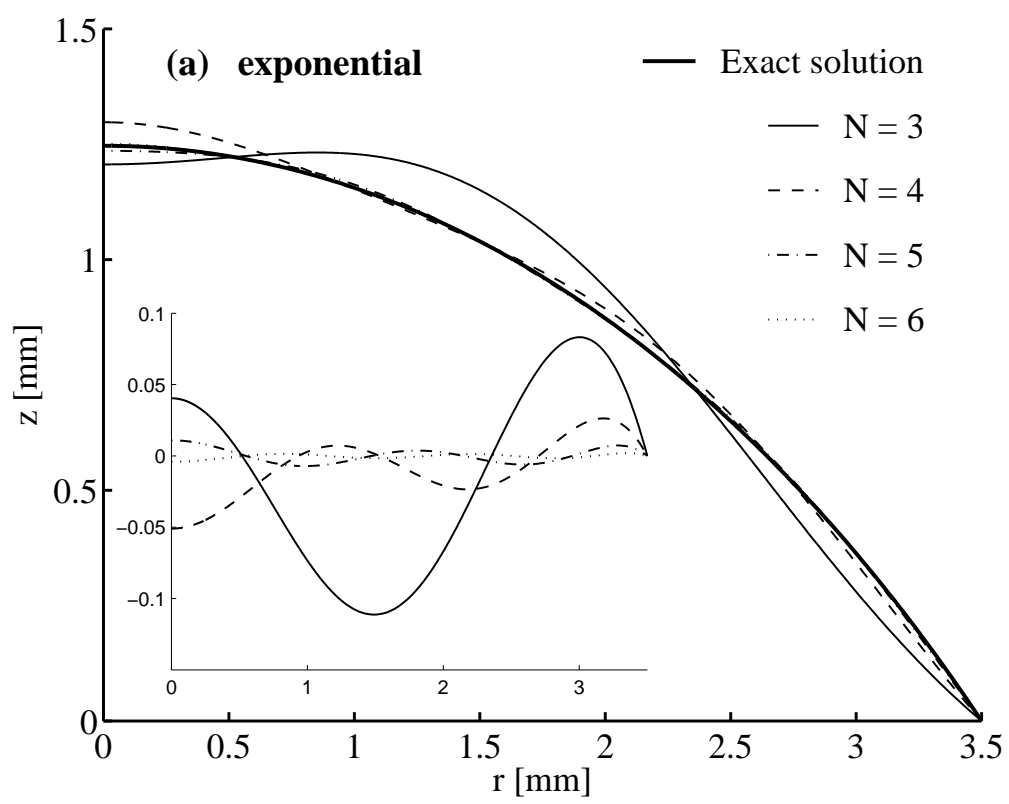

FiguRE 2: Convergence of Rayleigh-Ritz drop profiles towards the exact solution (spherical cap, thick solid line), for increasing amount of correction terms, $N$. Profiles are obtained with the exponential basis functions, (10). The inset shows the difference $z_{\mathrm{sph}}(r)-z_{\mathrm{RR}}(r)$. Physical parameters: $g \Delta \rho=$ $0, \gamma=72.8 \mathrm{mN} / \mathrm{m}, V_{0}=25 \mu \mathrm{L}, r_{c}=3.5 \mathrm{~mm}$.

where $n=3, \ldots, N$. Varying the drop volume does not modify our results phenomenologically; the volume is therefore kept constant throughout this discussion.

For an unstressed drop, Figure 2 shows the profiles obtained with the Rayleigh-Ritz method, for increasing amount of correction terms, using the exponential basis functions defined in (10). The inset is a plot of the difference between exact and approximated drop profiles, $z_{\mathrm{sph}}(r)-z_{\mathrm{RR}}(r)$, where $z_{\mathrm{RR}}(r)$ is the Rayleigh-Ritz solution (4). For all choices of basis functions, profiles obtained with only one term $(N=3)$ are in relative poor agreement 

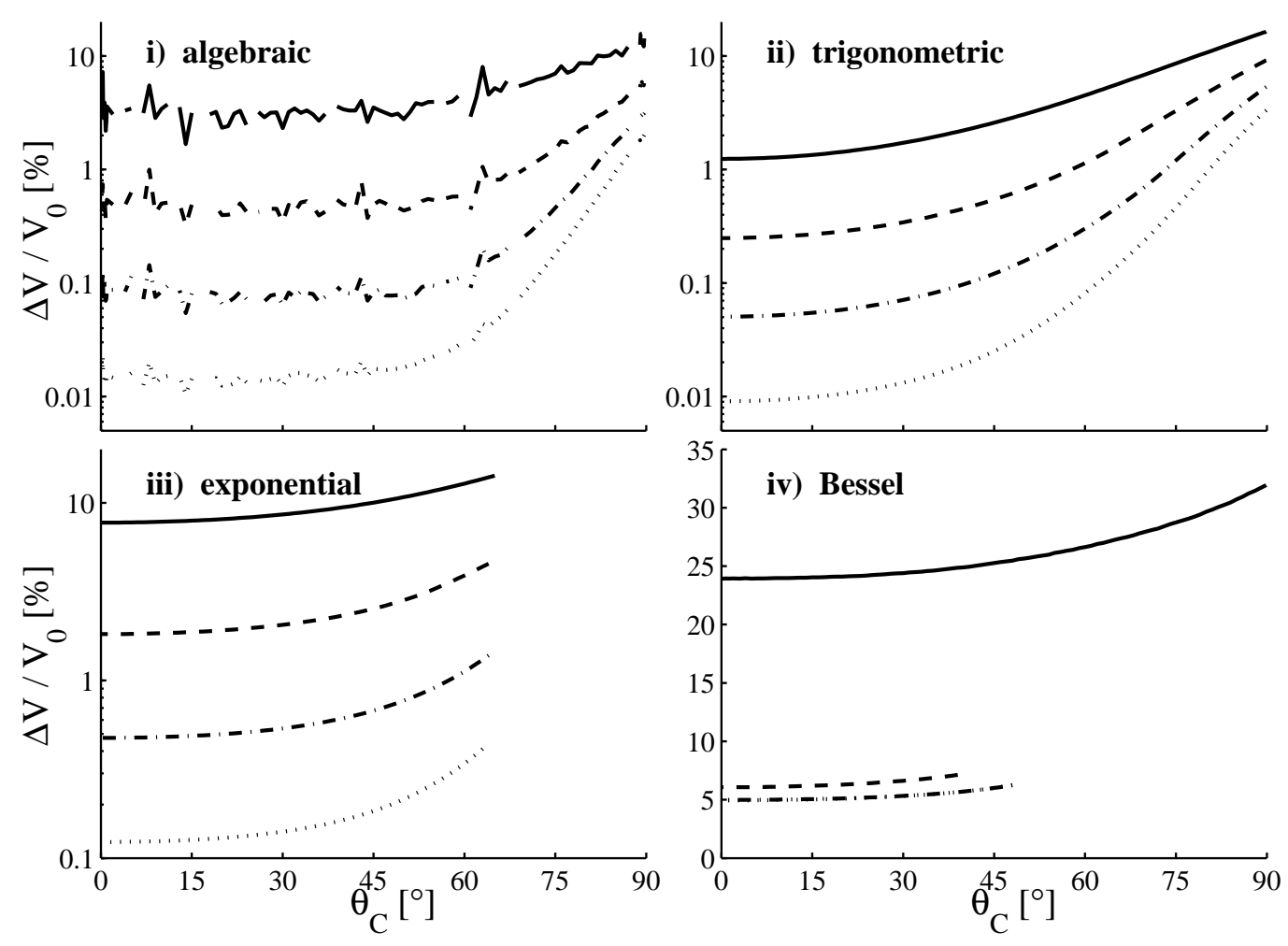

FiguRE 3: Accuracy of the Rayleigh-Ritz solutions, for all basis functions (10), and increasing amount of terms. The accuracy is characterized by the volume $\Delta V$, given in percentage of the drop volume, $V_{0}$. Significance of the lines and physical parameters like in Figure 2. 
with the exact solution (11). As more terms are added, the series converge towards the sphere section, with the exception of the Bessel function expansion that seems to converge toward a different profile.

The rate of convergence and accuracy of the solutions depend strongly on the choice of basis function $f_{n}(r)$. The accuracy of the Rayleigh-Ritz drop profiles are characterized by the volume enclosed between the exact and the numerical shape,

$$
\Delta V:=2 \pi \int_{0}^{r_{c}} r\left|z_{\mathrm{sph}}(r)-z_{\mathrm{RR}}(r)\right| d r .
$$

For all basis functions (10), Figure 3 illustrates the accuracy of the RayleighRitz profiles, in terms of the ratio $\Delta V / V_{0}$, as function of the number of correction terms, $N$, and the contact angle, $\theta_{c}$. For a prescribed drop volume and contact radius, the contact angle of the exact solution, $\theta_{c}$, is given by $\cos \theta_{c}=1-z_{0} / R$ (see Figure $1 \mathrm{~b}$ ). With all basis functions, the accuracy of the Rayleigh-Ritz solutions increases as the drop contact radius, $r_{c}$, increases, that is, for decreasing contact angles, $\theta_{c}$. Similarly, as the number of terms increases we get better profiles as measured by a decreasing ratio $\Delta V / V_{0}$.

Clearly, for any value of the contact radius, the Bessel function basis, $f_{n}(r)=J_{0}\left(\alpha_{0 n} r / r_{c}\right)$, does not give a good representation of the spherical profile, no matter how many terms are included in the series (see Figure 3iv). As we extend the number of expansion coefficients, the Rayleigh-Ritz solutions converge toward a limiting profile definitely different from the exact solution. The volume bounded by the Rayleigh-Ritz predictions and the spherical cap is relatively large compared with the corresponding values found with other basis functions. In addition, the values of the coefficients $\left\{a_{n}\right\}_{n=3}^{N}$ increase rapidly with increasing number of terms, giving rise to problems in the program for rounding the profile shape (note the incomplete lines in Figure 3 -iv), for $N \geqslant 4$ and $\theta_{c} \gtrsim 45^{\circ}$.

Exponential basis functions, $f_{n}(r)=\frac{1}{n} \exp \left[-n\left(r / r_{c}\right)^{2}\right]$, generate profiles that slowly converge towards the exact solution, see Figure 2). Using at least 

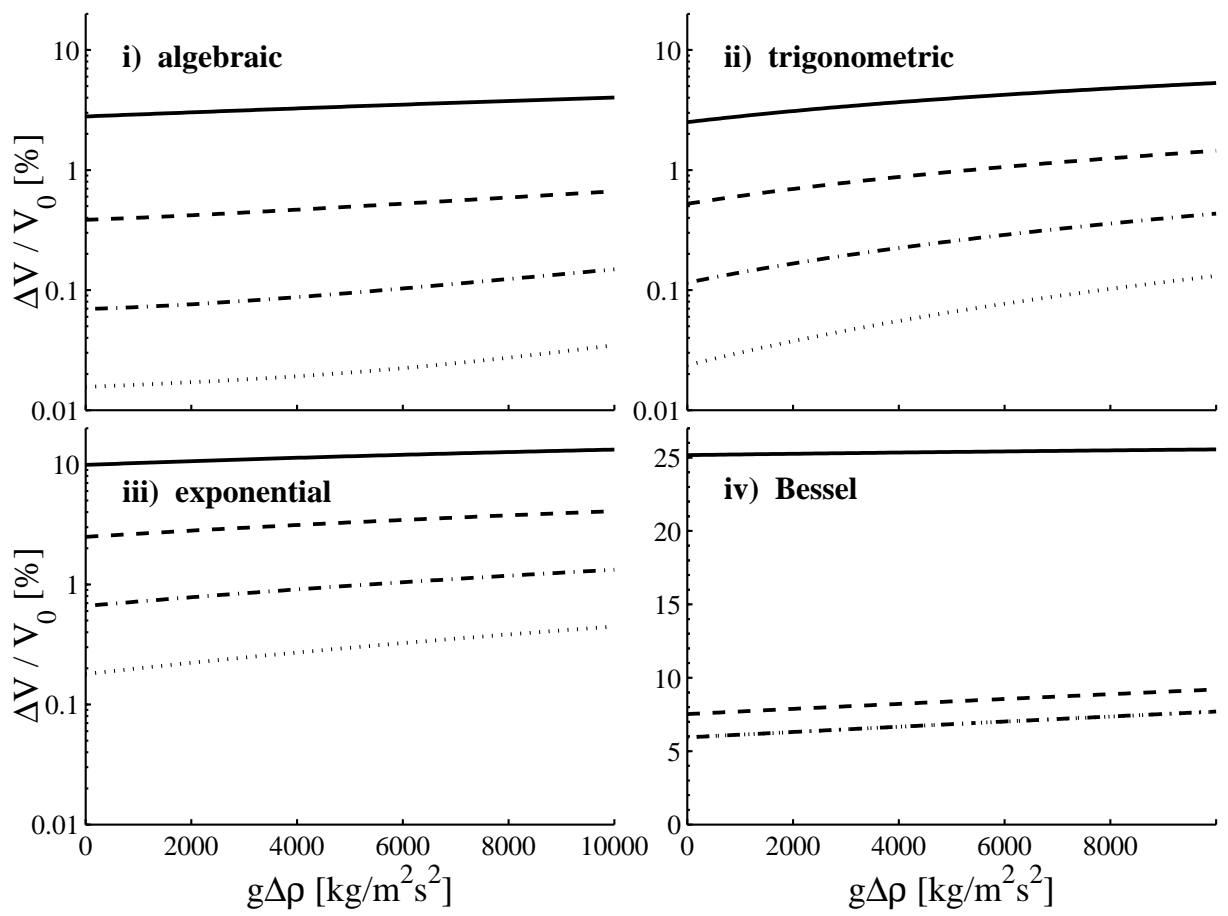

FiguRE 4: Accuracy of the Rayleigh-Ritz solutions for drops under gravitational stress. Significance of the lines like in Figures 2-3. Physical parameters are $\gamma=72.8 \mathrm{mN} / \mathrm{m}, V_{0}=100 \mu \mathrm{L}, r_{c}=5.3 \mathrm{~mm}$.

four terms, the ratio $\Delta V / V_{0}$ is of order $0.1 \%$, see Figure 3-iii). However, this basis suffers the same drawback as the Bessel function basis: for small contact radii $\left(\theta_{c}>65^{\circ}\right)$, the Rayleigh-Ritz coefficients become very large and numerical problems appear.

For drops of small to medium contact radii $\left(\theta_{c} \gtrsim 35^{\circ}\right)$, the algebraic basis functions, $f_{n}(r)=r_{c}^{2 n}-r^{2 n}$, give the most satisfying results. These rapidly produce accurate profiles compared with the sphere cap solution. With four or more terms, $\Delta V / V_{0} \simeq 0.01 \%$ for low contact angle values. Large coefficients, however, are generated for some contact radii, while for 

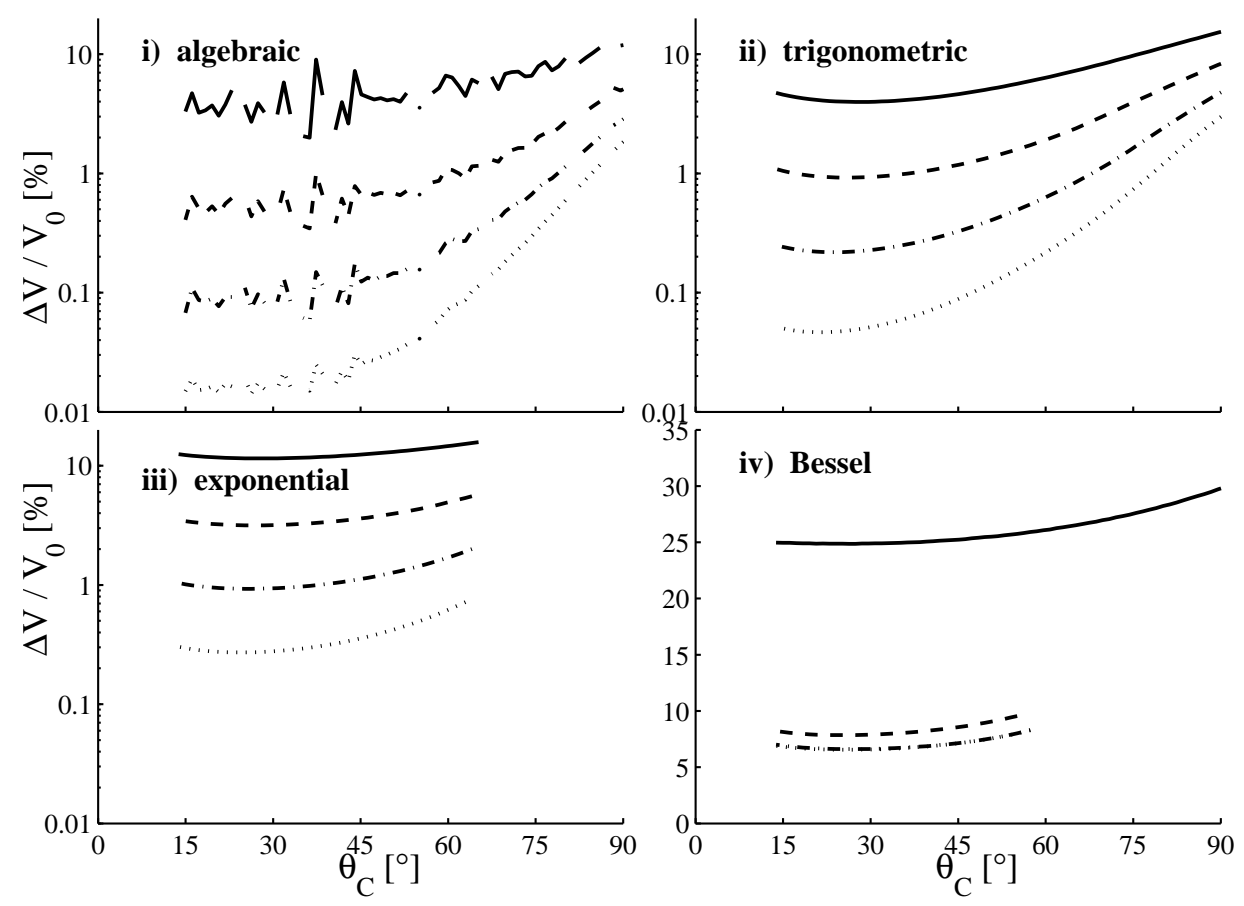

FiguRE 5: Accuracy of the Rayleigh-Ritz solutions for drops under gravitational stress. Significance of the lines like in Figures 2-3. Physical parameters are $g \Delta \rho=9776 \mathrm{~kg} / \mathrm{m}^{2} \mathrm{~s}^{2}$ (right). 
some values of $r_{c}$ no solution can be found at all, no matter how many terms are included in the series (see the "gaps" in the curves in Figure 3-i).

The trigonometric basis functions, $f_{n}(r)=\cos \left(\frac{1}{2} \pi n r / r_{c}\right)$, produce better profiles with increasing number of terms. Indeed, for large contact radii, these are the most satisfying functions: the ratio $\Delta V / V_{0}$ drops below $0.01 \%$ for small values of $\theta_{c}$, when taking at least four terms into account. This function choice incidentally produces small, $\left\{a_{n}\right\}_{n=3}^{N}$ values, that decrease with increasing number of terms, avoiding numerical complications. This makes this choice of basis a strong candidate.

\subsection{A sessile drop stressed under gravity}

For this system we set up the same scenario as in the previous section. The only change we make to the system is to include a gravitation contribution. That is, a finite gravity, $g$, and density difference, $\Delta \rho$, across the liquidgas interface. The physical parameters and fixed values for this system are $g=9.81 \mathrm{~m} / \mathrm{s}^{2}, \gamma=72.8 \mathrm{mN} / \mathrm{m}, V_{0}=100 \mu \mathrm{L}$ and $\mathcal{A}=0$, since we assume no interaction force. The prescribed drop radius, $r_{c}$, and density difference, $\Delta \rho$, are the variables we vary in this case, as well as the number of correction terms, $a_{n}$, where $n=3, \ldots, N$. Variation of the volume does not lead to qualitative modifications of our results.

With a gravitational force, increases in density difference result in increasingly flatter drop at the apex. This effect is observed for density differences $\Delta \rho>10 \mathrm{~kg} / \mathrm{m}^{3}$. The profile shape under a gravitational force is expected to be below the spherical shape, a fact confirmed using a sufficient number of terms. Due to the volume condition, successive terms tend to push the profile out at the edges while making the drop flatter at the apex, as a result of the influence of gravity.

For a given density difference, $\Delta \rho$, the convergence of the Rayleigh-Ritz solutions towards the exact solution exhibits similar overall behavior as for 

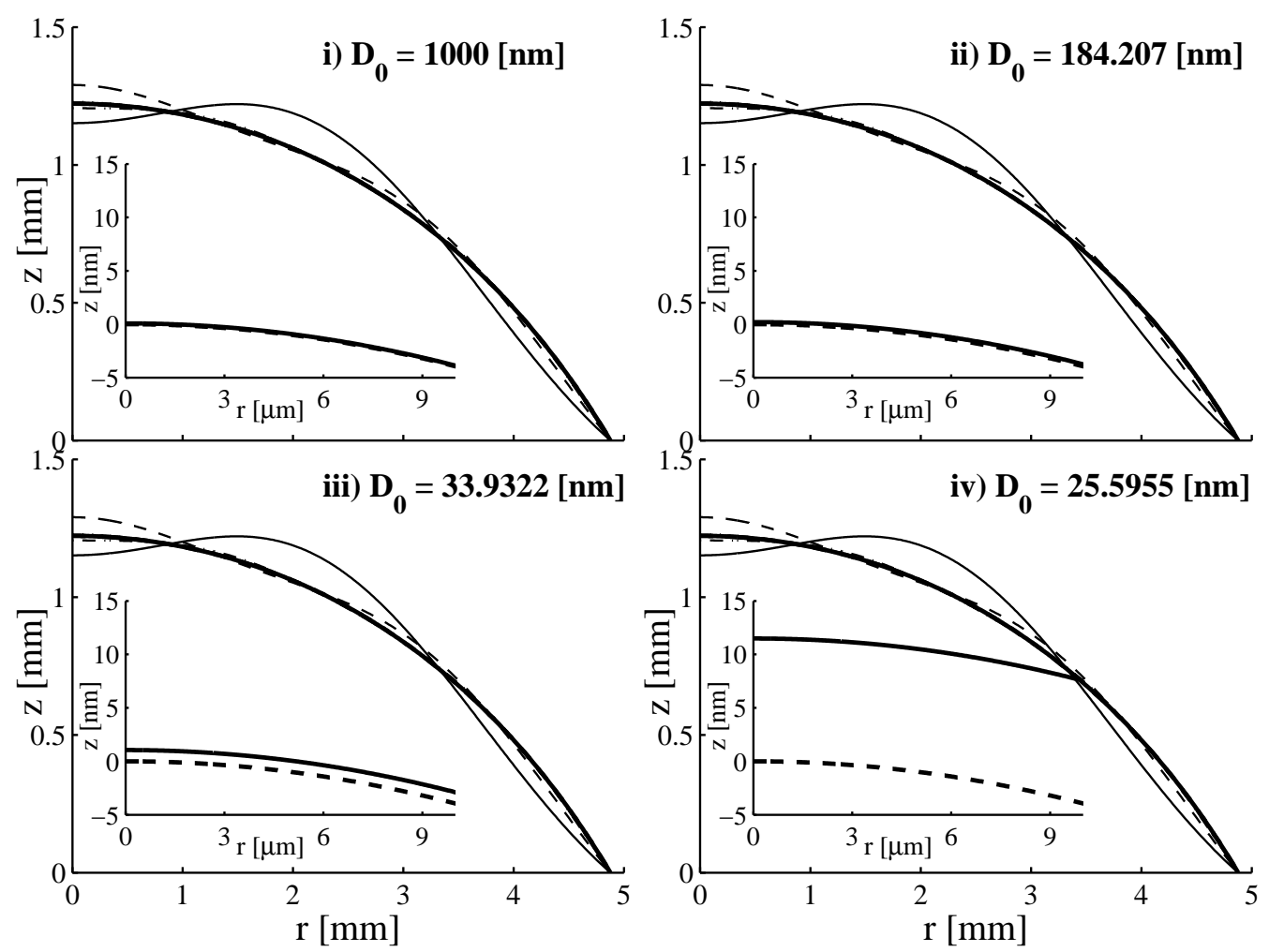

FiguRE 6: Effect of the van der Waals attraction on a water drop profile, as the interacting particle approaches. Position of the particle given by the separating distance at the apex, $D_{0}$. The insets show the separation around the apex between exact and the interaction-free solutions. Rayleigh-Ritz profiles obtained with the exponential function form. Physical parameters: $\mathcal{A}=20 \cdot 10^{-21} \mathrm{~J}, R_{p}=250 \mu \mathrm{m}$. Significance of the lines like in Figures 2-3. 

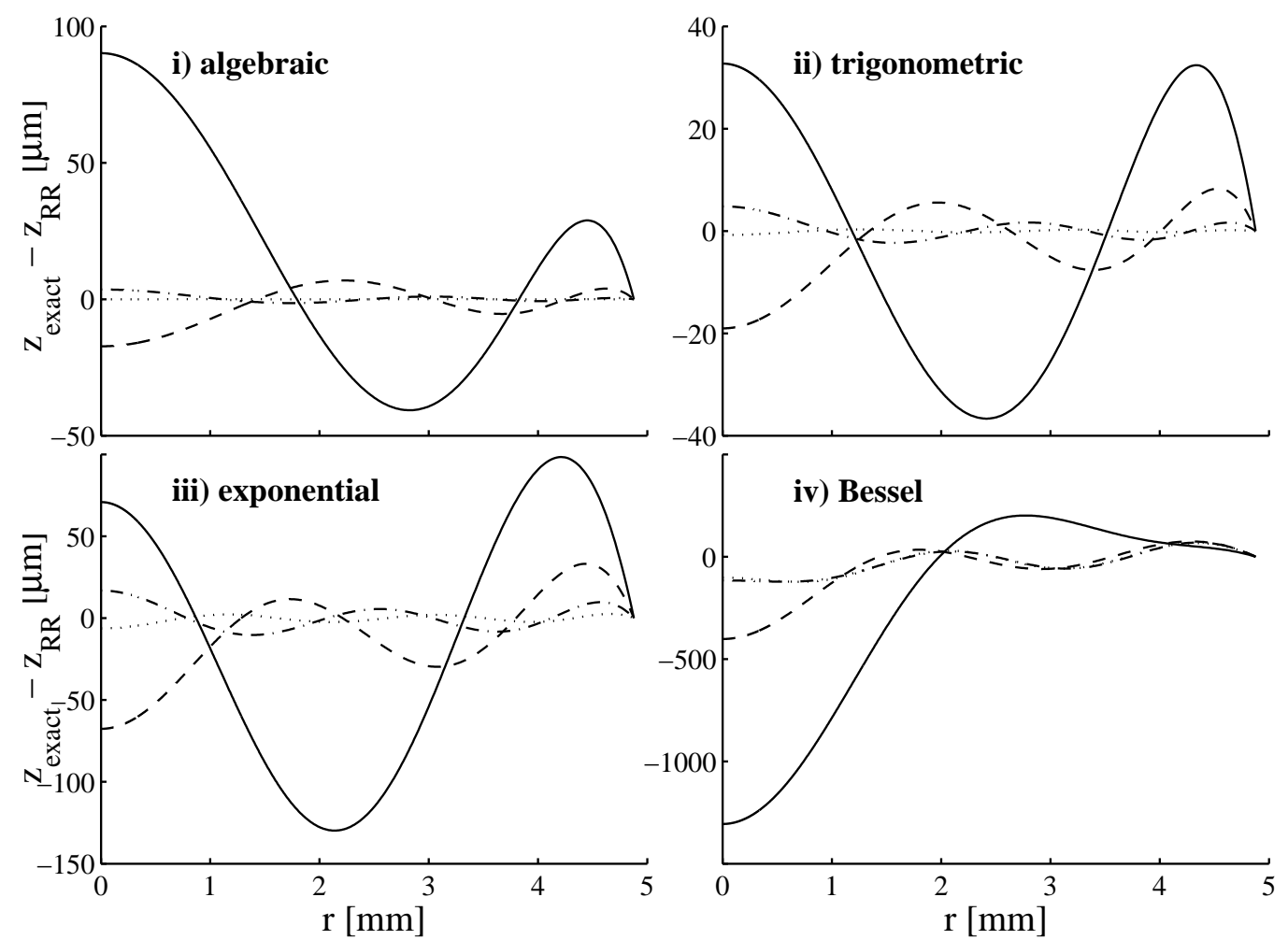

Figure 7: Difference between the Rayleigh-Ritz and the exact solutions, for drops set to combined gravitational and van der Waals stress. Physical parameters: $\mathcal{A}=10 \cdot 10^{-21} \mathrm{~J}, R_{p}=100 \mu \mathrm{m}, D_{0}=20 \mathrm{~nm}$. Significance of the lines like in Figures $2-3$. 
the unstressed drop. However, to obtain a satisfying solution, more correction terms are necessary because of the additional complication of the gravitational body force. This is confirmed by a study of the accuracy, as a function of the contact angle and the density difference, see Figures 4-5. In terms of the contact angle dependence, the accuracy of the RayleighRitz solutions is best with the algebraic basis for low contact angles and the trigonometric function basis for large contact angles. For a fixed contact radius, values of the ratio $\Delta V / V_{0}$ increase with the gravitational factor $g \Delta \rho$, implying that, as the effect of gravity increases, more correction terms must be incorporated to the series to obtain a given level of accuracy. Note that in the presence of gravity, for all choices of basis functions considered and any number of correction terms, the Rayleigh-Ritz solutions have the best accuracy for drops with a certain contact angle $\left(\theta_{c} \approx 25^{\circ}\right.$ in the case illustrated in Figures 4-5). This value for the optimal contact angle decreases with diminishing gravitational effect, and becomes $0^{\circ}$ in absence of gravitational stress.

Rayleigh-Ritz solutions are numerically difficult to obtain under the same conditions as for unstressed drops (see previous subsection); only the trigonometric function basis is always reliable. On the other hand, determination of the exact solution by numerical integration of the differential equation (2) is troublesome when the contact angle is too small. As a consequence, we have no exact solutions for drops with $\theta_{c}<15^{\circ}$.

\subsection{A sessile drop stressed under gravity and interaction}

In this section we consider a drop in air, under the combined influence of gravity and an attractive van der Waals interaction. The physical parameters and fixed values for this system are $g=9.81 \mathrm{~m} / \mathrm{s}^{2}, \Delta \rho=997 \mathrm{~kg} / \mathrm{m}^{3}, \gamma=$ $72.8 \mathrm{mN} / \mathrm{m}, V_{0}=50 \mu \mathrm{L}$ and $r_{c}=4.9 \mathrm{~mm}$. The spherical particle radius, $R_{p}$, the Hamaker constant, $\mathcal{A}$, and the minimum separation between particle 
and drop, $D_{0}$, are the variables we vary in this case. We gradually increase the interaction constant, $\mathcal{A}$, and the particle radius, $R_{p}$, to see if and how it affects the profile shape of the drop and where the extreme limits for the minimum separating distance, $D_{0}$, are to be found. The differences in profiles, $z_{\text {exact }}(r)-z_{\mathrm{RR}}(r)$, shown in Figure 7 indicate how small the deformations are for various minimum distances $D_{0}$.

The van der Waals attraction is maximal at the drop apex, but affects the whole drop shape. Under an attractive stress, the drop profile is raised up around the apex, and pushed in around the edges. When the particle approaches the drop surface, the apex deformation grows at an exponential rate. However, the interacting particle cannot be brought arbitrarily close to the drop surface: below a certain limiting separation, denoted $D_{0, \text { min }}$, the particle is too close and triggers rupture of the interface. That point corresponds to the stability limit of the system, where the drop 'jumps up' and captures the particle. At larger separations, the drop shape differs only marginally from the solution without interaction. A significant difference is only be observed when the particle is close to the stability limit.

The deformation process for decreasing apex separations, $D_{0}$, is shown in Figure 6. The thick solid lines are exact solutions, and the thick dashed line is the profile in the absence of interaction $(\mathcal{A}=0$, gravity only). As seen from the insets, the extent of the deformation due to the van der Waals stress is very small in comparison with the deformation induced by gravity. Around the apex, the profile rise is of the order of nanometers. The Rayleigh-Ritz solutions obtained with the exponential basis functions are also plotted in Figure 6, for the first four correction terms. At the apex, the magnitude of these corrections is much larger than the extent of the van der Waals deformation itself. For a fixed separation close to the limit, $D_{0, \text { min }}$, we show in Figure 7 the convergence of Rayleigh-Ritz profiles as a function of number of terms. For the bases considered, it is necessary to include at least 10 terms in the Rayleigh-Ritz series in order to reach the level of detail typical of the van der Waals deformation. The incorporation of a fair number of correction 

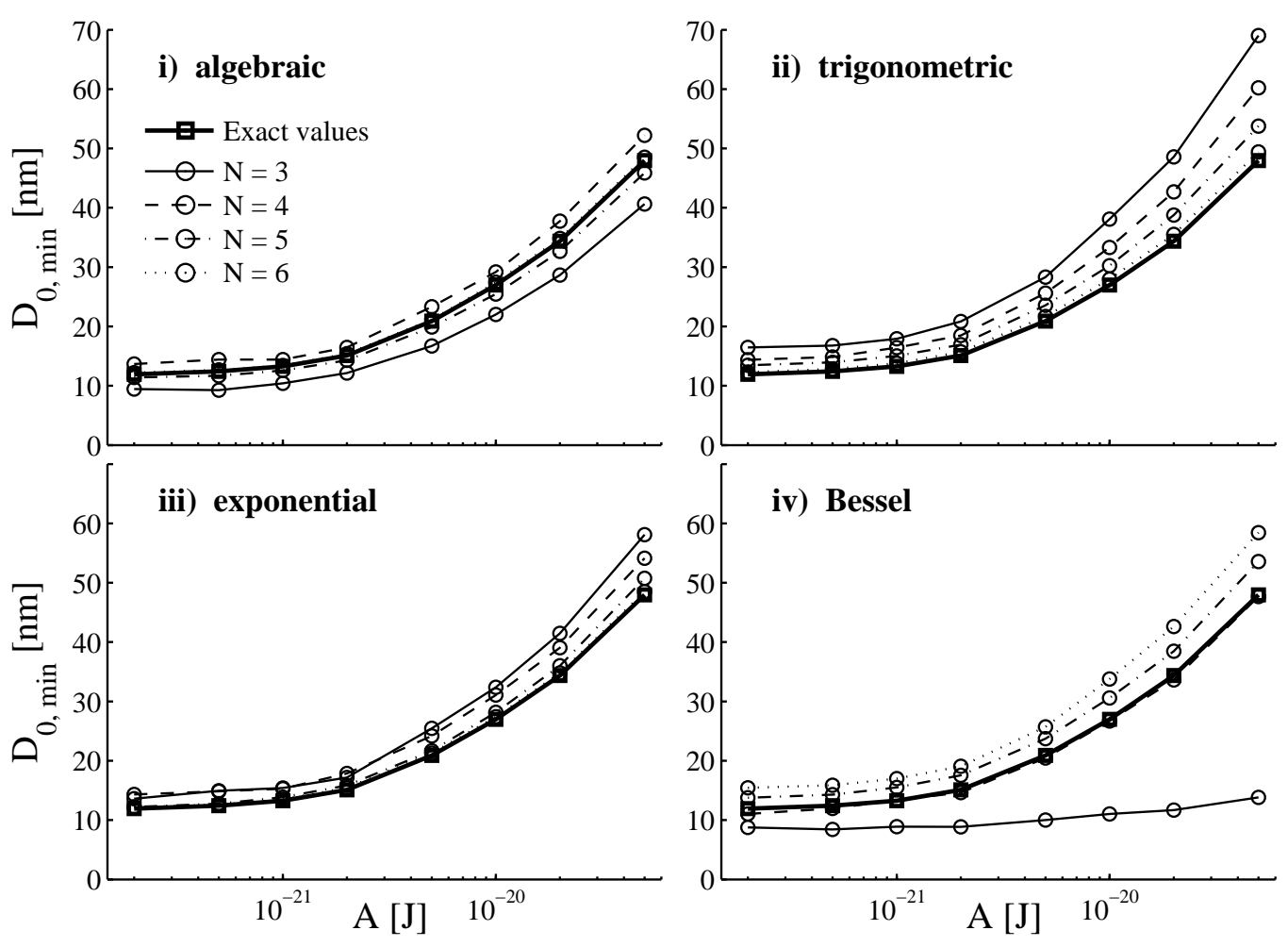

Figure 8: Determination of the closet approach distance, $D_{0, \min }$, using the Rayleigh-Ritz profiles, for different values of the Hamaker constant, $\mathcal{A}$, and particle radius, $R_{p}$. Physical parameters are $R_{p}=500 \mu \mathrm{m}$. 

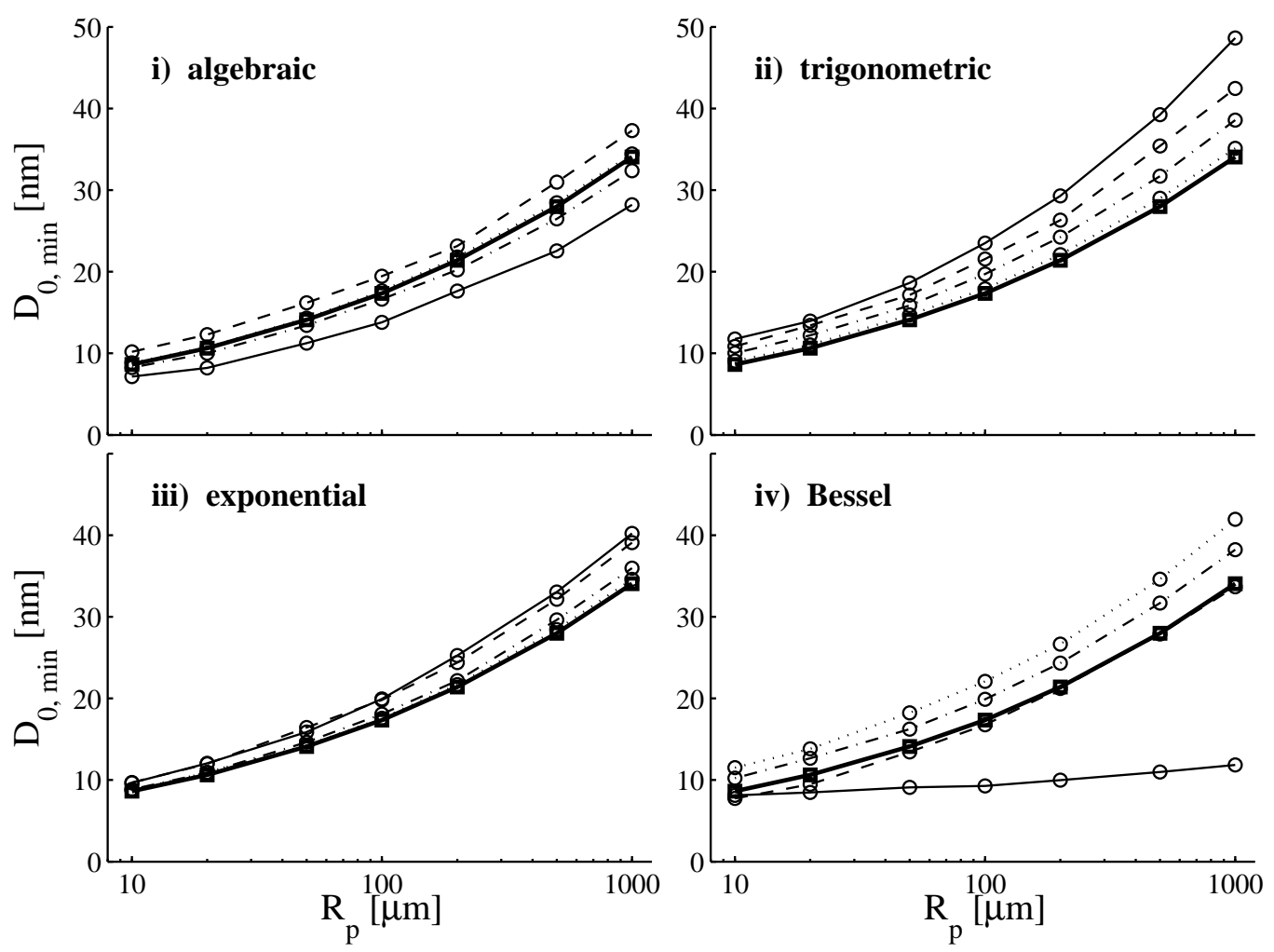

Figure 9: Determination of the closet approach distance, $D_{0, \min }$, using the Rayleigh-Ritz profiles, for different values of the Hamaker constant, $\mathcal{A}$, and particle radius, $R_{p}$. Physical parameters are $\mathcal{A}=10^{-21} \mathrm{~J}$. 
terms is thus necessary to obtain a solution that reliably reproduces the effect of the interaction around the apex.

The small deformation and its slow growth upon particle approach lead to drop shapes very close to those obtained for a simple gravitational stress. Even for large particle radii, $R_{p}$, or large Hamaker constants, $\mathcal{A}$, we observe small interaction induced profile differences. If sufficiently large, the drop radius, $r_{c}$, and density difference, $\Delta \rho$, do make the drop more compact and stable. This gives rise to small profile absolute changes due to interaction. Since the particle draws up the drop surface by means of the attractive interaction, increasing the density difference, $\Delta \rho$, results in smaller profile changes and gives rise to more squat, heavier and stable drops.

In varying the separation $D_{0}$, the Rayleigh-Ritz solutions and the ratio $\Delta V / V_{0}$ remain almost constant. The accuracy is reasonable and comparable to those for drops set under gravity only. However, in contrast with the discussion of Figures 6-7, accuracy in terms of the whole, macroscopic drop volume, $V_{0}$, is not an appropriate measure considering the small scale of the van der Waals deformation.

For values of $D_{0}$ below the stability limit, $D_{0, \text { min }}$, numerical problems appear and it is in principle impossible to find Rayleigh-Ritz solutions. Using this as a criterion to detect the limit, in Figures 8-9 we compare the limiting separations, $D_{0, \text { min }}$, found for the Rayleigh-Ritz solution, with the limits for the full numerical solution of (2)

Physically, the minimum distance of closest approach, $D_{0, \text { min }}$, becomes smaller for decreasing particle radius and Hamaker constant. That is, a small, weakly interacting spherical particle can be placed closer to the surface of the drop without causing the drop surface to rupture. We may find that we may need to increase the particle radius or Hamaker constant in order to induce a sufficient interaction to significantly affect the drop surface.

The limits predicted by use of the Rayleigh-Ritz solutions depend unfortunately on the number of terms, $N$. However, the physical trends, in 
term of dependence on Hamaker constant and particle radius, are respected. Moreover, with the notable exception of the Bessel function basis using only one term, we obtain values of $D_{0, \min }$ that closely agree with the exact limiting separation. It is one of our aims in future work to improve the detection of the stability limit.

\section{Overall summary}

The Rayleigh-Ritz method obtains approximate solutions for a sessile drop under various conditions of stress. We made use of an ansatz that takes care of the basic conditions of substrate contact and constant volume condition, as well as the boundary condition of constant contact radius. The solutions were compared with full numerical solutions of the Euler-lagrange equation, (2), and characterized in terms basis function choice and number of terms. In general, the macroscopic accuracy is very good with just a few correction terms, and the Rayleigh-Ritz method provides a fast and reliable way of determining drop shapes. For systems including interaction with a particle, more correction terms must by taken into account to capture the small deformation induced by the van der Waals attraction; only then are solutions accurate at the scale characteristic of the interaction. The limiting separation, $D_{0, \min }$, can also be determined for any particle geometry and interaction strength. The results are, in general, in good agreement with the physical behavior and accurate numerical limiting values.

\section{References}

[1] H.-J. Butt, J. Colloid Interface Sci. 166, 109 (1994). C777

[2] W. A. Ducker, Z. Xu and J. N. Israelachvili, Langmuir 10, 3279 (1994). C777 
[3] M. L. Fielden, R. A. Hayes and J. Ralston, Langmuir 12, 3721 (1996). C777

[4] S. Basu and M. M. Sharma, J. Colloid Interface Sci. 181, 443 (1996). C777

[5] P. G. Hartley, F. Grieser, P. Mulvaney and G. W. Stevens, Langmuir 15, 7282 (1999). C777

[6] S. A. Nespolo, D. Y. C. Chan, F. Grieser, P. G. Hartley and G. W. Stevens, Langmuir 19, 2124 (2003). C777

[7] R. G. Horn, D. J. Bachmann, J. N. Connor and S. J. Miklavcic, J. Phys.: Condens. Matter 8, 9483 (1996). C777

[8] J. N. Connor, Measurement of Interactions Between Solid and Fluid Surfaces, Doctoral Thesis: University of South Australia, 2001, ISBN 0-868039152. C777

[9] J. N. Connor, and R. G. Horn, Langmuir 17, 7194 (2001). C777

[10] J. N. Connor and R. G. Horn, Rev. Sci. Inst. 74, 4601 (2003). C777

[11] N. Chen, T. Kuhl, R. Tadmor, Q. Lin and J. Israelachvili, Phys. Rev. Lett. 92, 024501:1-4 (2004). C777

[12] M. L. Forcada, N. R. Arista, A. Gras-Marti, H. M. Urbassek, and R. Garcia-Molina, Phys. Rev. B 44, 8226 (1991). C777

[13] S. J. Miklavcic, R. G. Horn and D. J. Bachmann J. Phys. Chem. 99, 16357 (1995). C777

[14] S. J. Miklavcic Phys. Rev. E 54, 561 (1996). C777

[15] D. Bachmann, and S. J. Miklavcic, Langmuir 12, 4197 (1996). C777

[16] S. J. Miklavcic Phys. Rev. E 57, 561 (1998). C777 
[17] D. Y. C. Chan, R. R. Dagastine and L. R. White J. Colloid Interface Sci. 236, 141 (2001). C777, C778

[18] D. E. Aston and J. C. Berg, J. Colloid Interface Sci. 235, 162 (2001). C777

[19] D. Bhatt, J. Newman and C. J. Radke, Langmuir 17, 116 (2001). C777

[20] S. J. Miklavcic and P. Attard, J. Phys. A: Math. Gen. 34, 7849 (2001). C777, C780

[21] P. Attard, and S. J. Miklavcic, Langmuir 17, 8217 (2001). C777, C778

[22] D. C. Bardos, Surface Science 517, 157 (2002). C777, C778

[23] R. Courant and D. Hilbert, Methods of Mathematical Physics (Interscience Publishers Inc., New York, 1989) Vol. 1. C778, C781 